\title{
Tensorial split-field finite-difference time-domain approach for second- and third-order nonlinear materials
}

\author{
Jorge Francés, ${ }^{1,2, *}$ Jani Tervo, ${ }^{3}$ and Andrés Márquez ${ }^{1,2}$ \\ ${ }^{1}$ Department of Physics, Systems Engineering and Signal Theory, University of Alicante, San Vicente del Raspeig Drive, \\ NW, Alicante, Ap. 99, E-3080, Spain \\ ${ }^{2}$ University Institute of Physics to Sciences and Technologies, University of Alicante, San Vicente del Raspeig Drive, \\ NW, Alicante, Ap. 99, E-3080, Spain \\ ${ }^{3}$ Department of Physics and Mathematics, University of Eastern Finland, P.O. Box 111, FI-80101 Joensuu, Finland \\ *Corresponding author: jfmonllor@ua.es
}

Received March 6, 2013; revised April 15, 2013; accepted April 27, 2013;

posted April 29, 2013 (Doc. ID 186477); published May 30, 2013

The split-field finite-difference time-domain (SF-FDTD) method for one-dimensionally periodic structures is
extended to include the coefficient-tensor description of second- and third-order nonlinear-optical media. A
set of nonlinear equations related to the split-field values of the electric field is established. An iterative
fixed-point approach for solving the coupled nonlinear system of equations needed to update the electric field
components in the SF-FDTD is then developed. The third-order nonlinear susceptibility dispersion is also con-
sidered by means of the Raman effect and its implementation in the SF-FDTD scheme. Different scenarios are
considered in order to verify the reliability of the method for simulating second- and third-order nonlinear-optical
media. First, second-harmonic generation and its efficiency are investigated in a homogeneous layer with and
without the quasi-phase-matching technique. Second, the nonlinear dispersion is analyzed by means of the
generation of solitons in Kerr media due to the Raman effect. Last, a set of binary phase gratings with nonlinear
pillars is considered under oblique incidence. Here the nonlinear refractive index is generated by different
physical mechanisms modeled with the nonscalar third-order susceptibility. @ 2013 Optical Society of America physical mechanisms modeled with the nonscalar third-order susceptibility. ( 2013 Optical Society of Ameri
OCIS codes: (050.1755) Computational electromagnetic methods; (050.1950) Diffraction gratings;

(190.4360) Nonlinear optics, devices.

http://dx.doi.org/10.1364/JOSAB.30.001711

\section{INTRODUCTION}

Nonlinear media have attracted a great deal of interest in the last few decades [1,2]. In real materials, nonlinear effects are typically weak, and hence a high incident intensity is often required in order to observe nonlinear-optical effects. However, in optical nanostructures, remarkably lower input intensities may be sufficient to trigger nonlinear effects due to high field confinement [1, $\underline{3}]$. In the last few years, nonlinear phenomena have been investigated, e.g., to study how to control light and to design all-optical ultrafast signal-processing devices such as waveguides combining linear and nonlinear media [4], all-optical switching devices [5,6], or periodic structures created from different nonlinear media [7].

Second-harmonic generation (SHG) has played an increasingly important role in several near-field optics applications such as the determination of surface magnetic and electric properties [8,9], rough surface studies [10], and apertureless scanning near-field microscopy [11]. Owing to the crucial role of light polarization in many integrated-optical devices, anisotropic properties of the nonlinear susceptibility must be taken into account. An example of this is the coupling of a TM polarized incident field into a TE polarized second-harmonic one. In third-order nonlinear materials, there is a cubic dependence between electric polarization and electric field, which implies that there is an intensity-dependent nonlinear contribution to the refractive index [1]. This characteristic in periodic structures gives rise to a wide range of phenomena that have been applied to achieve new photonics applications such as bistability [12], the zero- $n$ gap based on alternating positive and negative index material [13-16], and integrated optoelectronics $[\underline{17}, \underline{18}]$.

In order to design even more complex optical devices, it is essential to have a time-efficient analysis tool. The initial finite-difference time-domain (FDTD) method [19] has been one of the most successful tools in calculating the time evolution of electromagnetic fields in many different types of structures. In the case of laterally periodic media, the FDTD technique can be modified to take full advantage of the periodicity of the problem. One such modification is the so-called periodic boundary condition (PBC). However, in the case of oblique illumination, the $\mathrm{PBC}$ requires a phase shift between the periods, which significantly reduces the performance of the approach.

The split-field finite-difference time-domain (SF-FDTD) approach [20-26] is a powerful method for analyzing periodic structures under oblique incidence, a situation in which the ordinary FDTD approach encounter difficulties. In SF-FDTD only one period is considered rather than the whole structure, which has obvious advantages as far as numerical efficiency is concerned. In particular, the outstanding studies 
by Shahmansouri and Rashidian have significantly improved the SF-FDTD approach $[27,28]$ by introducing the possibility of analyzing dispersive media.

Even though the state of the art in classical FDTD formulation also covers nonlinear media [29-35], the split-field approach must be thoroughly reformulated in such cases. In this paper, an extension of the SF-FDTD approach for certain second- and third-order nonlinear materials with tensorial nonlinear susceptibilities is presented. In SF-FDTD for nonlinear periodic media, the Floquet theorem is taken into account as in the corresponding linear case. Furthermore, the nonlinear dispersion of the third-order susceptibility is also included in the formulation. The improvement is based on a recent paper by the authors focusing on the SF-FDTD implementation of the electro-optical and all-optical Kerr effect in periodic media [36]. Since the materials are nonlinear, a coupled nonlinear system of equations must be solved in SF-FDTD. Here, the iterative fixed-point procedure, discussed in detail in [36], is applied to analyze a wide range of situations such as the SHG under quasi-phase matching (QPM), the nonlinear dispersion (Raman effect) in Kerr media, and different physical mechanisms that lead to a nonlinear refractive index in third-order nonlinear materials.

This paper is organized as follows. First, we present the theory for the split-field formulation with second-order nonlinear media and SHG. Second, we describe how the scheme is extended to cover third-order nonlinear media, beginning with the nonlinear dispersion behavior and concluding with the tensorial formulation of the susceptibility. Third, we present numerical examples for various types of structures in order to illustrate the potential of this approach.

\section{SPLIT-FIELD FINITE-DIFFERENCE TIME-DOMAIN METHOD}

In this section, we present the basis for the SF-FDTD method and the implementation of the different extensions. Figure 1(a) shows the basic geometry considered throughout the paper. We assume that the structure is periodic in the $x$ direction, and that the input light field is a plane wave such that the wave vector $\mathbf{k}_{\text {inc }}$ forms an angle $\theta_{0}$ with the positive $z$ axis. The periodicity of the problem is taken into account by applying the $\mathrm{PBC}$ at the periodic boundaries. To truncate the problem in the $z$ direction, we apply the uniaxial perfectly matched layer (UPML) method $[\underline{37,38}]$ (UPML). The input plane-wave source is excited in the structure following the schemes proposed in $[\underline{29}, \underline{24}]$ for both continuous and time-limited pulsed waves. Our approach is restricted to one-dimensional periodic structures, but an extension to twodimensional periodic structures is expected to be straightforward (cf. the split-field formulation for linear media in [27]). The distribution of the field components within one unit cell is illustrated in Fig. 1(b).

\section{A. Basic Concepts}

To facilitate the derivation of the new formulation, we next recall the derivation of SF-FDTD from earlier studies. Here we assume the media are nonmagnetic and nonconducting, and hence Maxwell's curl equations take on the form

$$
\begin{gathered}
\nabla \times \mathbf{E}=-\mu_{0} \frac{\partial \mathbf{H}}{\partial t}, \\
\nabla \times \mathbf{H}=\frac{\partial \mathbf{D}}{\partial t},
\end{gathered}
$$

where $\mu_{0}$ is the permeability of free space, $\mathbf{D}$ is the electric displacement vector in the space-time domain, and $\mathbf{H}$ is the magnetic field. We split the electric displacement vector into linear and nonlinear parts with

$$
\mathbf{D}=\epsilon_{0} \epsilon_{r} \mathbf{E}+\mathbf{F}^{\mathrm{NL}},
$$

where $\epsilon_{0}$ is the vacuum permittivity, $\epsilon_{r}$ denotes the relative linear permittivity, and $\mathbf{F}^{\mathrm{NL}}$ is the nonlinear polarization. Consequently, Eq. (2) can be rewritten as

$$
\nabla \times \mathbf{H}=\epsilon_{0} \epsilon_{r} \frac{\partial \mathbf{E}}{\partial t}+\mathbf{J}^{\mathrm{NL}}
$$

where $\mathbf{J}^{\mathrm{NL}}$ is the nonlinear-polarization current density defined by $\partial \mathbf{F}^{\mathrm{NL}} / \partial t$.

Since we assume the material is illuminated by an obliquely incident plane wave and that the structure is periodic in the $x$ direction, the field everywhere contains a linear $x$-dependent phase term $\exp \left(j k_{x} x\right)$, where $k_{x}=(\omega / c) \sin \theta_{0}$ is the $x$ component of the wave vector in the phasor domain, $c$ is the vacuum speed of light, and $\omega$ is the angular frequency. In the SF formulation, we consider the split-field variables that eliminate the effect of the phase term using the transformation

$$
\breve{\mathbf{P}}=\breve{\mathbf{E}} e^{j k_{x} x}
$$

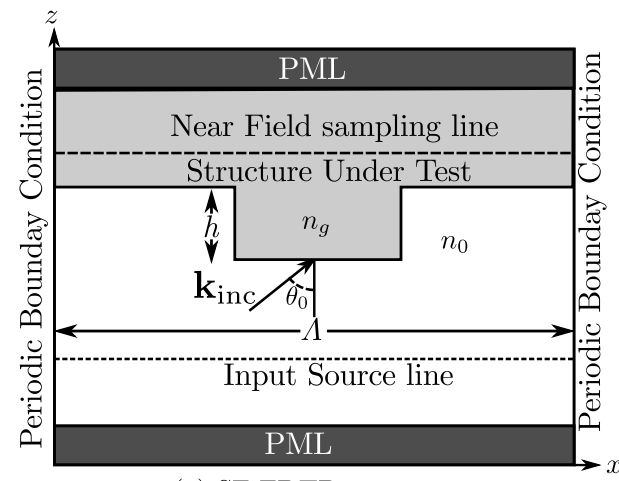

(a) SF-FDTD geometry

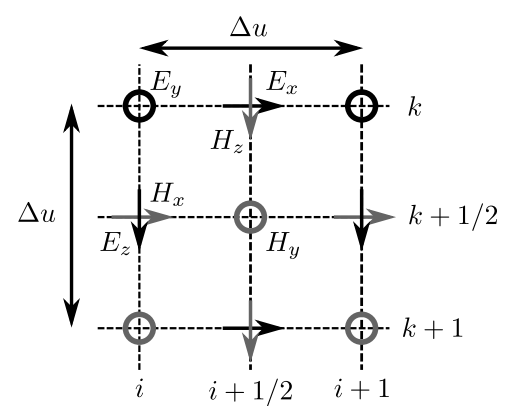

(b) Two-dimensional SF-FDTD grid

Fig. 1. (a) Scheme of a two-dimensional computational space. (b) Yee cell for a two-dimensional SF-FDTD scheme. 


$$
\breve{\mathbf{Q}}=\mu_{0} c \breve{\mathbf{H}} e^{j k_{x} x},
$$

where $\breve{\mathbf{P}}$ and $\breve{\mathbf{Q}}$ are the transformed vectors in the phasor domain. Analogous transformation can also be applied to the nonlinear-polarization current [36].

Substituting the split-field components into Maxwell's equations, performing some algebraic operations [36], and eliminating the time-derivative terms $\partial / \partial t \Leftrightarrow j \omega$, the basis for the SF-FDTD with nonlinear-polarization terms can be expressed as follows:

$$
\begin{gathered}
P_{x}=P_{x a}-c^{2} \mu_{0} \kappa F_{x}^{\mathrm{NL}} e^{j k_{x} x} \\
P_{y}=P_{y a}+\sin \theta_{0} \kappa Q_{z}-c^{2} \mu_{0} \kappa F_{y}^{\mathrm{NL}} e^{j k_{x} x}, \\
P_{z}=P_{z a}-\sin \theta_{0} \kappa Q_{y}-c^{2} \mu_{0} \kappa F_{z}^{\mathrm{NL}} e^{j k_{x} x}, \\
Q_{x}=Q_{x a} \\
Q_{y}=Q_{y a}-\sin \theta_{0} P_{z} \\
Q_{z}=Q_{z a}+\sin \theta_{0} P_{y}
\end{gathered}
$$

where the " $a$ " field components are fully detailed in [36]. In the following section, we discuss the implementation of the polarization terms in Eqs. (7)-(9) for each specific scenario.

\section{B. Second-Order Nonlinear Media}

It is well known that second-order nonlinear effects exist in various noncentrosymmetric optical crystals. In such a case, the polarization $\mathbf{F}$ can be expressed as a function of the second-order nonlinear susceptibility $\chi_{0}^{(2)}$ and the propagating electric field components inside the structure. The nonlinear response of the material leads to an exchange of energy between the fundamental field at frequency $\omega_{f}$ and the secondharmonic field at the double frequency $\omega_{s}=2 \omega_{f}$. In this process the induced nonlinear-polarization term is defined in terms of a third rank tensor $\mathbf{d}$ that can be represented in a contracted form as a $3 \times 6$ matrix $d_{\mu \mathrm{m}}$ :

$$
\mathbf{d}=\left[\begin{array}{llllll}
d_{11} & d_{12} & d_{13} & d_{14} & d_{15} & d_{16} \\
d_{21} & d_{22} & d_{23} & d_{24} & d_{25} & d_{26} \\
d_{31} & d_{32} & d_{33} & d_{34} & d_{35} & d_{36}
\end{array}\right]
$$

Thus, using this contracted suffix notation, the polarization term related to SHG can be defined by [으늘

$$
\begin{gathered}
F_{m}^{\mathrm{NL}, \omega_{f}}=2 \epsilon_{0} \sum_{\sigma, \beta} d_{m \xi} E_{\sigma}^{\omega_{f}} E_{\beta}^{\omega_{s}}, \\
F_{m}^{\mathrm{NL}, \omega_{s}}=\epsilon_{0} \sum_{\sigma, \beta} d_{m \xi} E_{\sigma}^{\omega_{f}} E_{\beta}^{\omega_{f}},
\end{gathered}
$$

where $m=1,2$, and 3 stand for $x, y$, and $z$, respectively. Suffix $\xi$ takes on values $1, \ldots 6$ that are related to the Cartesian axes by the rules given in Table $\underline{1}[\underline{1,}, \underline{39}]$.
Table 1. Contracted Matrix Notation for $d_{m \xi}$ Indices

\begin{tabular}{ccccccc}
\hline$\xi$ & 1 & 2 & 3 & 4 & 5 & 6 \\
\hline \multirow{2}{*}{$\sigma \beta$} & $x x$ & $y y$ & $z z$ & $z y$ & $z x$ & $x y$ \\
& & & & $y z$ & $x z$ & $y x$ \\
\hline
\end{tabular}

Taking into account the transformation into the split-field domain, we find that

$$
\left[\begin{array}{c}
F_{x}^{\mathrm{NL}, \omega_{f}} \\
F_{y}^{\mathrm{NL}, \omega_{f}} \\
F_{z}^{\mathrm{NL}, \omega_{f}}
\end{array}\right] e^{j \omega_{f} \sin \theta_{0} x / c}=2 \mathbf{d} \epsilon_{0}\left[\begin{array}{c}
P_{x}^{\omega_{f}} E_{x}^{\omega_{s}} \\
P_{y}^{\omega_{f}} E_{y}^{\omega_{s}} \\
P_{z}^{\omega_{f}} E_{z}^{\omega_{s}} \\
P_{z}^{\omega_{f}} E_{y}^{\omega_{s}}+P_{y}^{\omega_{f}} E_{z}^{\omega_{s}} \\
P_{z}^{\omega_{f}} E_{x}^{\omega_{s}}+P_{x}^{\omega_{f}} E_{z}^{\omega_{s}} \\
P_{x}^{\omega_{f}} E_{y}^{\omega_{s}}+P_{y}^{\omega_{f}} E_{x}^{\omega_{s}}
\end{array}\right],
$$

and

$$
\left[\begin{array}{l}
F_{x}^{\mathrm{NL}, \omega_{s}} \\
F_{y}^{\mathrm{NL}, \omega_{s}} \\
F_{z}^{\mathrm{NL}, \omega_{s}}
\end{array}\right] e^{j \omega_{s} \sin \theta_{0} x / c}=\mathbf{d} \epsilon_{0}\left[\begin{array}{c}
P_{x}^{\omega_{f}} P_{x}^{\omega_{f}} \\
P_{y}^{\omega_{f}} P_{y}^{\omega_{f}} \\
P_{z}^{\omega_{f}} P_{z}^{\omega_{f}} \\
2 P_{z}^{\omega_{f}} P_{y}^{\omega_{f}} \\
2 P_{z}^{\omega_{f}} P_{x}^{\omega_{f}} \\
2 P_{x}^{\omega_{f}} P_{y}^{\omega_{f}}
\end{array}\right]
$$

If we consider a two-dimensional crystal material with cubic symmetry, which belongs to class $23[1,39]$ with crystal axes matching the principal axes, the only nonzero elements in the coefficient tensor are $d_{14}=d_{25}=d_{36}$. This scheme is suitable for modeling GaAs-based structures, for instance.

We may now write Maxwell's equations for the fundamental and the second-harmonic split fields as

$$
\begin{gathered}
P_{x}^{\omega_{f}}=P_{x a}^{\omega_{f}}-2 d_{14} \kappa P_{z}^{\omega_{f}} E_{y}^{\omega_{s}}, \\
P_{z}^{\omega_{f}}=P_{z a}^{\omega_{f}}-\sin \theta_{0} \kappa Q_{y}^{\omega_{f}}-2 d_{14} \kappa P_{x}^{\omega_{f}} E_{y}^{\omega_{s}} \\
Q_{y}^{\omega_{f}}=Q_{y a}^{\omega_{f}}-\sin \theta_{0} P_{z}^{\omega_{f}}
\end{gathered}
$$

and

$$
\begin{gathered}
P_{y}^{\omega_{s}}=P_{y a}^{\omega_{s}}+\sin \theta_{0} \kappa Q_{z}^{\omega_{s}}-2 d_{14} \kappa P_{x}^{\omega_{f}} P_{z}^{\omega_{f}}, \\
Q_{x}^{\omega_{s}}=Q_{x a}^{\omega_{s}}, \\
Q_{z}^{\omega_{s}}=Q_{z a}^{\omega_{s}}+\sin \theta_{0} P_{y}^{\omega_{s}},
\end{gathered}
$$

respectively. Again, the " $a$ " fields in Eqs. (18)-(23) are defined in [36]. The nonlinear-polarization terms require averaging over the nearest nodes surrounding $P_{m}$ [see Fig. 1(b)]. For example, in Eq. (18), the values of $P_{z}^{\omega_{f}}$ and $E_{y}^{\omega_{s}}$ must be computed by means of the semi-implicit approximation as 
$(1 / 4)\left(\left.P_{z}^{\omega_{f}}\right|_{i, k+1 / 2} ^{e}+\left.P_{z}^{\omega_{f}}\right|_{i+1, k+1 / 2} ^{e}+\left.P_{z}^{\omega_{f}}\right|_{i, k-1 / 2} ^{e}+\left.P_{z}^{\omega_{f}}\right|_{k+1, k-1 / 2} ^{e}\right)$ and $(1 / 2)\left(\left.E_{y}^{\omega_{s}}\right|_{i, k} ^{e}+\left.E_{y}^{\omega_{s}}\right|_{i+1, k} ^{e}\right)$, respectively [24,43]. This approximation assumes that the field component is simply the arithmetic average of the stored values in the neighboring cells. Equations (18), (19), and (21) make up a system of equations of the form $\mathbf{P}=\mathbf{U}(\mathbf{P})$ that is solved by a fixed-point iterative process. This approach is based on solving the $p$ th equation in the original system for $P_{m}$. The next step is to choose the initial guess $\mathbf{P}^{(0)}$, after which we compute the subsequent iterations by

$$
\mathbf{P}^{(p+1)}=\mathbf{U}\left(\mathbf{P}^{(p)}\right), \quad p=0,1,2, \ldots
$$

In this specific case we found empirically that 15 iterations are required to obtain sufficient accuracy. However, we fixed the number of iterations far beyond this value in order to ensure the results converged. For example, with 25 iterations the results are very accurate and the computational time is not dramatically affected. The analysis of the convergence condition for fixed-point iterative process and FDTD can be found in $[36,44]$.

\section{Dispersive Media with Third-Order Nonlinearity}

In this section, we describe the extension of the SF-FDTD scheme for third-order nonlinearity with dispersion. In such a case, the third-order nonlinear polarization can be defined as a linear superposition of the form [45]

$$
\mathbf{F}^{\mathrm{NL}}=\mathbf{F}^{\mathrm{K}}+\mathbf{F}^{\mathrm{R}}
$$

where $\mathbf{F}^{\mathrm{K}}$ is the Kerr nonlinearity contribution and $\mathbf{F}^{\mathrm{R}}$ is the nonlinear dispersion due to the Raman effect. These two terms are fully detailed in the literature (cf. nonlinear media and the standard FDTD approach $[31,32, \underline{45}])$. The definition of the Kerr nonlinearity contribution is

$$
\mathbf{F}^{\mathrm{K}}(t)=\alpha \epsilon_{0} \chi_{0}^{(3)}|\mathbf{E}|^{2} \mathbf{E},
$$

where $\chi_{0}^{(3)}$ is the scalar nonlinear coefficient and $\alpha$ is a factor that parametrizes the relative strengths of the Kerr and Raman interactions.

The polarization due to the Raman effect is given by

$$
\mathbf{F}^{\mathrm{R}}(t)=\epsilon_{0} \mathbf{E}\left[\chi_{\text {Raman }}^{(3)} *|\mathbf{E}|^{2}\right],
$$

where $*$ denotes the convolution and $\chi_{\text {Raman }}^{(3)}$, fully defined in [45], accounts for nonresonant third-order processes, including phonon interactions and nonresonant electronic effects. We introduce an auxiliary variable $\mathbf{S}$ to facilitate the handling of the Raman model in SF-FDTD. After some mathematical operations, detailed in [45], and taking into account that $|\mathbf{E}|^{2}=|\mathbf{P}|^{2}$, we obtain the equation for updating $\mathbf{S}$ :

$$
\begin{aligned}
\mathbf{S}^{e+1 / 2}= & \frac{2-\left(\omega_{R} \Delta t / 2\right)^{2}}{1+\gamma_{R} \Delta t / 2} \mathbf{S}^{e}+\frac{\gamma_{R} \Delta t / 2-1}{1+\gamma_{R} \Delta t / 2} \mathbf{S}^{e-1 / 2} \\
& +\frac{(1-\alpha) \chi_{0}^{(3)}\left(\Delta t \omega_{R} / 2\right)^{2}}{1+\gamma_{R} \Delta t / 2}\left|\mathbf{P}^{e}\right|^{2}
\end{aligned}
$$

where $e$ stands for the step and

$$
\omega_{R}=\sqrt{\frac{\tau_{1}^{2}+\tau_{2}^{2}}{\tau_{1}^{2} \tau_{2}^{2}}} ; \quad \gamma_{R}=\frac{1}{\tau_{2}} .
$$

The time resolution of SF-FDTD is denoted by $\Delta t$, and $\tau_{1}$ and $\tau_{2}$ are chosen to fit the Raman-gain spectrum [31,32, $\left.\underline{45}\right]$.

We may next apply the field transformation to Eq. (27), which gives us

$$
\mathbf{F}_{R}^{e+1} e^{j k_{x} x}=\epsilon_{0} \mathbf{S}^{e+1} \mathbf{P}^{e+1}
$$

This term can now be included in Eqs. ()-(9) analogously to the Kerr currents in [36]

$$
\begin{gathered}
P_{x}=P_{x a}-\kappa\left(\alpha \chi_{0}^{(3)}\left|P_{x}\right|^{2} P_{x}+S_{x} P_{x}\right), \\
P_{y}=P_{y a}+\sin \theta_{\mathrm{inc}} \kappa Q_{z}-\kappa\left(\alpha \chi_{0}^{(3)}\left|P_{y}\right|^{2} P_{y}+S_{y} P_{y}\right), \\
P_{z}=P_{z a}-\sin \theta_{\mathrm{inc}} \kappa Q_{y}-\kappa\left(\alpha \chi_{0}^{(3)}\left|P_{z}\right|^{2} P_{z}+S_{z} P_{z}\right) .
\end{gathered}
$$

Equations (31)-(33) form a nonlinear system of equations and hence, when updating the SF-FDTD equations, we need to employ an iterative process in each time step. Here we choose to use the fixed-point iteration, detailed in [36]. In this case, the main equation for the fixed-point iteration is essentially similar to the one used with the Kerr model. However, here the update coefficients $C_{m}$ that appear in [36] are substituted by new update coefficients that include both the Kerr and the Raman effects.

The update step of the total fields given in Eqs. (7)-(12) can be reformulated removing the dependencies properly:

$$
\begin{gathered}
P_{z}=\frac{P_{z a}-\kappa \sin \theta_{0} Q_{y a}}{1+\kappa\left(\chi_{0}^{(3)}\left|E_{z}\right|^{2}+S_{z}-\sin ^{2} \theta_{0}\right)}=C_{z} \hat{P}_{z a}, \\
Q_{y}=Q_{y a}-\sin \theta_{0} P_{z},
\end{gathered}
$$

$$
P_{x}=\frac{P_{x a}}{1+\kappa\left(\chi_{0}^{(3)}\left|E_{x}\right|^{2}+S_{x}\right)}=C_{x} P_{x a},
$$

$$
\begin{gathered}
P_{y}=\frac{P_{y a}+\kappa \sin \theta_{0} Q_{z a}}{1+\kappa\left(\chi_{0}^{(3)}\left|E_{y}\right|^{2}+S_{y}-\sin ^{2} \theta_{0}\right)}=C_{y} \hat{P}_{y a}, \\
Q_{z}=Q_{z a}+\sin \theta_{0} P_{y},
\end{gathered}
$$

where $C_{m}$ are defined by

$$
\begin{gathered}
C_{x}=\frac{1}{1+\kappa\left(\chi_{0}^{(3)} I_{x}+S_{x}\right)}, \\
C_{y}=\frac{1}{1+\kappa\left(\chi_{0}^{(3)} I_{y}+S_{y}-\sin ^{2} \theta_{0}\right)},
\end{gathered}
$$




$$
C_{z}=\frac{1}{1+\kappa\left(\chi_{0}^{(3)} I_{z}+S_{z}-\sin ^{2} \theta_{0}\right)},
$$

and

$$
\mathbf{I}=|\mathbf{E}|^{2} .
$$

The general equation for the fixed-point iteration is $\left.\mathbf{I}\right|_{p+1}=\left.\mathbf{P}\right|_{p}\left(\left.\mathbf{P}\right|_{p}\right)^{*}$, which can be extended for each space coordinate

$$
\begin{aligned}
& \left.I_{x}\right|_{p+1}=\left.C_{x}\right|_{p}\left(\left.C_{x}\right|_{p}\right)^{*}\left|P_{x a}\right|^{2}, \\
& \left.I_{y}\right|_{p+1}=\left.C_{y}\right|_{p}\left(\left.C_{y}\right|_{p}\right)^{*}\left|\hat{P}_{y a}\right|^{2}, \\
& \left.I_{z}\right|_{p+1}=\left.C_{z}\right|_{p}\left(\left.C_{z}\right|_{p}\right)^{*}\left|\hat{P}_{z a}\right|^{2},
\end{aligned}
$$

where

$$
\begin{gathered}
\left.C_{x}\right|_{p}=\frac{1}{1+\kappa\left(\left.\chi_{0}^{(3)} I_{x}\right|_{p}+S_{x}\right)}, \\
\left.C_{y}\right|_{p}=\frac{1}{1+\kappa\left(\left.\chi_{0}^{(3)} I_{y}\right|_{p}+S_{y}-\sin ^{2} \theta_{0}\right)},
\end{gathered}
$$

(a)

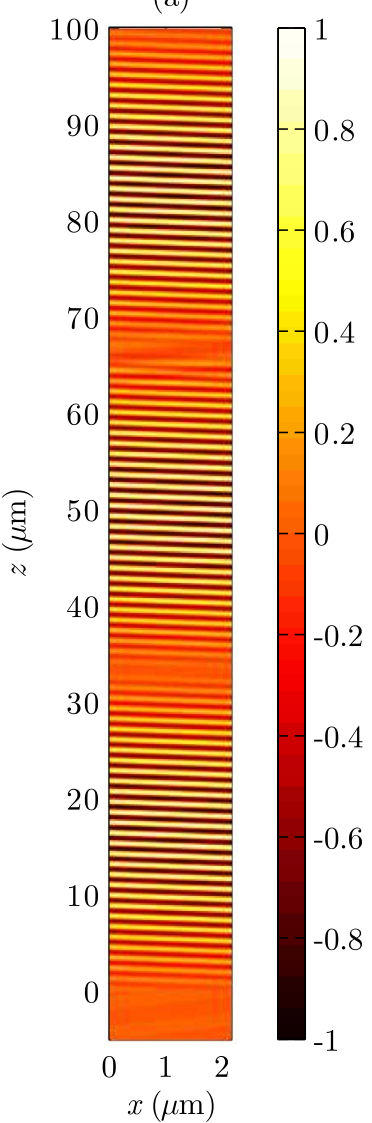

$$
\left.C_{z}\right|_{p}=\frac{1}{1+\kappa\left(\left.\chi_{0}^{(3)} I_{z}\right|_{p}+S_{z}-\sin ^{2} \theta_{0}\right)}
$$

are updated in each iteration.

It is worth noting that here the subindex $p$ is an integer related to the iteration step for the fixed-point iteration process, and not for the spatial dimension. The updating process performed by the fixed-point iterations takes up most of the computation time in SF-FDTD calculations. Therefore, this process should be avoided whenever both Kerr and Raman effects can be disregarded. Regarding convergence, the fixed-point iteration establishes an inequality that depends on the nonlinear parameters, and also on the input-field amplitude. This expression can easily be derived from [36] by adding the Raman contribution. An in-depth analysis of this issue can be found in [44].

\section{Tensorial Description of Third-Order Nonlinear Media}

In the preceding analysis, the nonlinear third-order effects were described by a scalar susceptibility $\chi^{(3)}$ value. However, the third-order susceptibility is a fourth-rank tensor, and it is characterized by 81 elements. In this section, we shall deal with isotropic media, in which case the number of independent elements can be reduced dramatically due to the spatial symmetry [39,46]. In particular, semiconductor materials such as silicon or GaAs, which are crystals belonging to class 32, have cubic symmetry, and hence their nonlinear

(b)
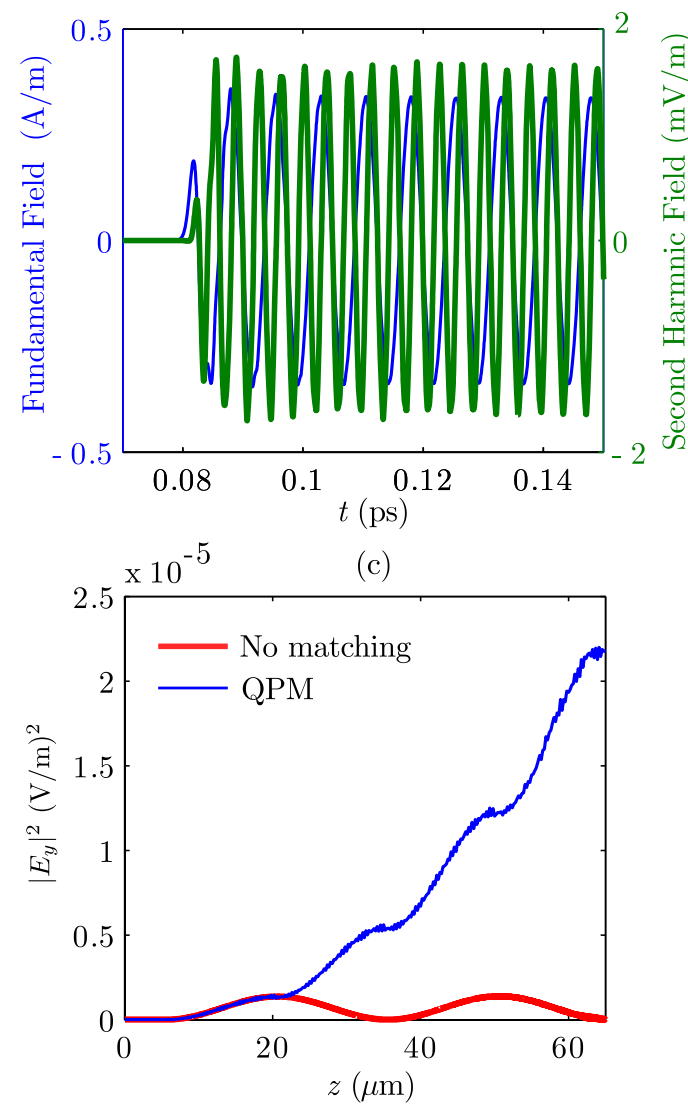

Fig. 2. (a) Second-harmonic field amplitude as a function of space. (b) Time-domain results for the fundamental (thin blue line) and secondharmonic (thick green line) fields at a point along the device at the center of the simulation grid. (c) Second-harmonic intensity along the nonlinear media with (thick red line) and without QPM (thin blue line). 
susceptibilities can be described by a tensor with only two independent components [1]. The relative magnitude of these parameters depends on the nature of the physical process behind the nonlinearity.

The nonlinear-polarization term for third-order materials with the physical characteristics mentioned below can be simplified to [1]

$$
\mathbf{F}^{\mathrm{NL}}=\epsilon_{0} A\left(\mathbf{E} \cdot \mathbf{E}^{*}\right) \mathbf{E}+\frac{1}{2} \epsilon_{0} B(\mathbf{E} \cdot \mathbf{E}) \mathbf{E}^{*}
$$

The ratio between $B$ and $A$ accounts for different physical mechanisms that lead to the nonlinear behavior of materials. In particular,

- $B / A=6$ for molecular orientation,

- $B / A=1$ for nonresonant electronic response,

- $B / A=0$ for electrostriction.

If we develop the polarization terms pertaining to the TM polarization in the split-field domain, we obtain the expressions

$$
\begin{aligned}
& F_{x}^{\mathrm{NL}}=\epsilon_{0}\left[\left(A+\frac{1}{2} B\right)\left|E_{x}\right|^{2} E_{x}+A\left|E_{z}\right|^{2} E_{x}+\frac{1}{2} B E_{z}^{2} E_{x}^{*}\right], \\
& F_{z}^{\mathrm{NL}}=\epsilon_{0}\left[\left(A+\frac{1}{2} B\right)\left|E_{z}\right|^{2} E_{z}+A\left|E_{x}\right|^{2} E_{z}+\frac{1}{2} B E_{x}^{2} E_{z}^{*}\right] .
\end{aligned}
$$

In Eqs. ( $\underline{50})$ and (1ㅣ), the terms with the squared field amplitude values can be written in the following form:

$$
\begin{aligned}
& E_{z}^{2} E_{x}^{*}=\left|E_{z}\right|^{2} e^{j 2 \Phi_{z}} \frac{E_{x}}{E_{x}} E_{x}^{*}=\left|E_{z}\right|^{2} E_{x} e^{-j 2 \Phi}, \\
& E_{x}^{2} E_{z}^{*}=\left|E_{x}\right|^{2} e^{j 2 \Phi_{x}} \frac{E_{z}}{E_{z}} E_{z}^{*}=\left|E_{x}\right|^{2} E_{z} e^{j 2 \Phi}
\end{aligned}
$$

with $\Phi(t)=\Phi_{x}(t)-\Phi_{z}(t)$ being the phase difference [46]. Equations (50) and (51) in the split-field domain yield

$$
\begin{aligned}
& F_{x}^{\mathrm{NL}} e^{j k_{x} x}=\epsilon_{0}\left[\left(A+\frac{1}{2} B\right)\left|P_{x}\right|^{2}+\left(A+\frac{1}{2} B e^{-j 2 \Phi}\right)\left|P_{z}\right|^{2}\right] P_{x} \\
& F_{z}^{\mathrm{NL}} e^{j k_{x} x}=\epsilon_{0}\left[\left(A+\frac{1}{2} B e^{j 2 \Phi}\right)\left|P_{x}\right|^{2}+\left(A+\frac{1}{2} B\right)\left|P_{z}\right|^{2}\right] P_{z}
\end{aligned}
$$

which can be substituted into Eqs. (7) and (9), respectively. This yields a new nonlinear system of equations that can also be solved by an iterative fixed-point procedure with $P_{x}$ and $P_{z}$ as unknowns, and Eqs. (7)-(9) as $\mathbf{U}(\mathbf{P})$.

\section{RESULTS}

\section{A. Second-Harmonic Generation}

We first consider a GaAs-based homogenous layer in order to analyze the SHG in nonlinear media. The excitation field is a continuous-wave TM signal at a fundamental wavelength of
$2.25 \mu \mathrm{m}, \theta_{0}=30 \mathrm{deg}$, and amplitude $12 \pi \Omega \mathrm{A} / \mathrm{m}$ in the splitfield domain $\left(132.63 \mathrm{~mW} / \mathrm{m}^{2}\right)$. For the sake of illustration, two matching scenarios are considered. First, no matching technique is used so the level of coupling between the input and the generated waves depends entirely on the phase shift between them. This phase shift is defined by the difference between the effective indices of the two propagating modes.

Second, a QPM based on a periodically poled material is assumed. A periodically poled material is a structure that is fabricated in such a manner that the orientation of one of the crystal axes is inverted periodically as a function of the position within the material [1]. Here it is assumed that the period $\Lambda$ of the alteration of the axis is equal to twice the coherent buildup length of the nonlinear interaction. This length can easily be identified visually in Fig. 2(c), where the efficiency of the second-harmonic wave without QPM has an oscillatory behavior. Figure 2(a) shows the increase and decrease in the second-harmonic amplitude $\left(E_{y}\right)$ as a function of the space without QPM. Figure 2(b) represents the timedomain transverse profiles of both fundamental and second harmonics at a point along the structure. The simulation verifies the coupling of second-harmonic energy on the first odd TE mode. The results clearly show the relative frequency between the two propagating waves.

\section{B. Dispersive Third-Order Nonlinear Materials}

Let us next study the results based on the analysis of dispersive third-order nonlinearity. In order to validate the method discussed here, the interaction of a pulsed optical source with a material having nonlinear dispersive properties is shown.

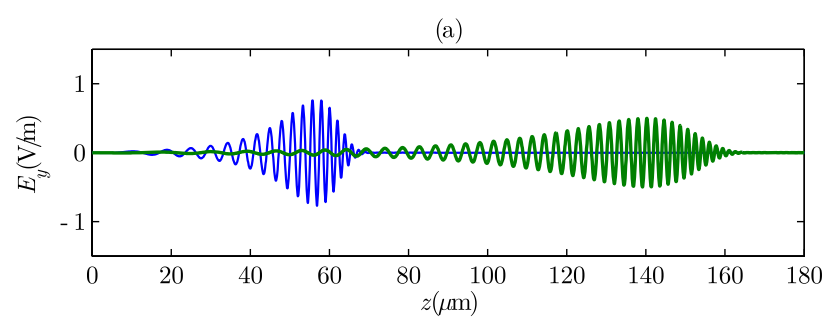

(b)

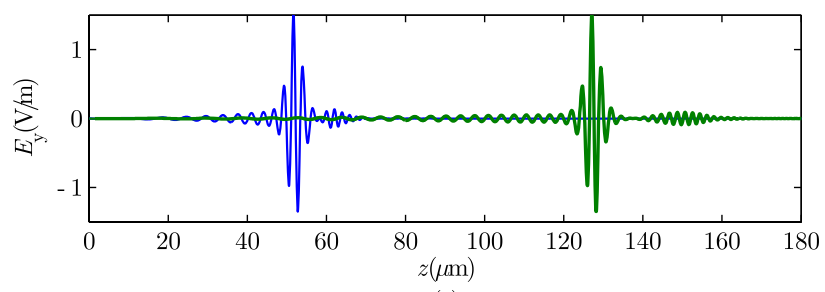

(c)

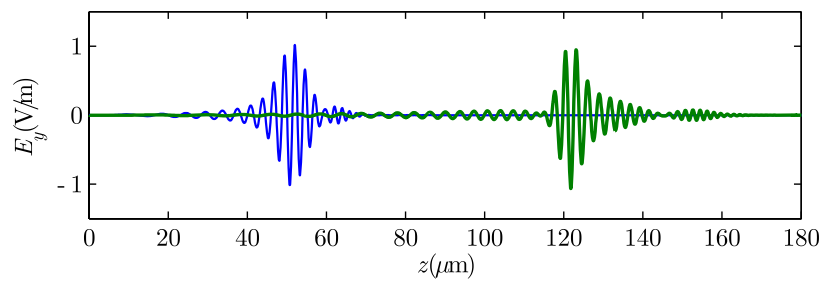

Fig. 3. (a) Snapshots of the electric field $\Re\left\{E_{y}\right\}$ in different kinds of media detected from left to right at times $t=2000 \Delta t$ (thin blue line) and $4000 \Delta t$ (thick green line), respectively. (a) Linear dispersive Lorentz medium. (b) Nonlinear Lorentz and Kerr media. (c) Nonlinear Lorentz, Kerr, and Raman media. 


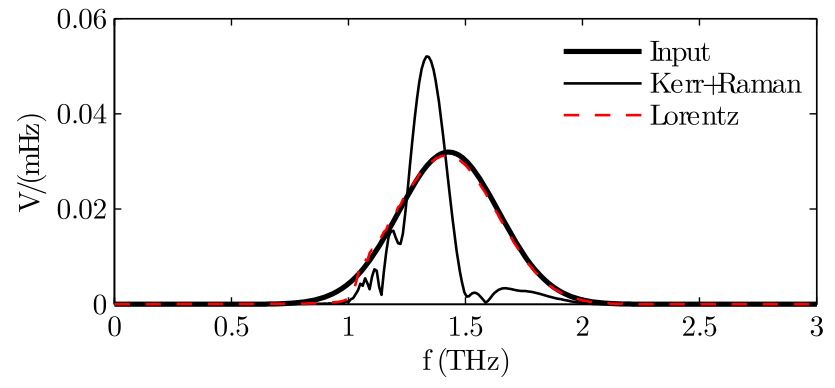

Fig. 4. Frequency spectrum for the case of a pulse propagating in nonlinear dispersive media. Sampling plane at $z=1500 \Delta$.

The optical pulse is switched on at $t=0$ at the surface $z=0$ of a material. The amplitude of the pulse has a maximum absolute value of $1.1 \mathrm{~V} / \mathrm{m}\left(80.24 \mathrm{~mW} / \mathrm{m}^{2}\right)$ with a carrier frequency $f_{c}=1.37 \times 10^{14} \mathrm{~Hz}(\lambda=2.19 \mu \mathrm{m})$. Here we consider an ideal model in which the whole pulse consists of three carrier cycles only. In this example, we fix the spatial resolution of the simulation at $52.5 \mathrm{~nm}\left(\approx \lambda_{0} / 40\right)$. In FDTD computations with nonlinear media, it is very common to scale both the susceptibility term and the input intensity to trigger the nonlinearity with lower input fields, thus reducing roundoff errors [29-32]. The dispersive characteristics are given by the following.

- Linear dispersion: $\epsilon_{s}=5.25, \quad \epsilon_{\infty}=2.25, \quad \omega_{L}=$ $4 \times 10^{14} \mathrm{~s}^{-1}, \gamma_{L}=2 \times 10^{9} \mathrm{~s}^{-1}$.
Table 2. Setup Parameters of SF-FDTD for Results in Figs. $\underline{5}$ and $\underline{6}$

\begin{tabular}{ccccccc}
\hline$\lambda_{0}(\mathrm{~nm})$ & $\Delta u(\mathrm{~m})$ & $\Delta t(\mathrm{~s})$ & $r_{x}($ cells $)$ & $r_{z}($ cells $)$ & $r_{\mathrm{PML}}($ cells $)$ & $e_{\text {steps }}$ \\
\hline 633 & $\lambda_{0} / 30$ & $\Delta u /\left(\sqrt{2} c_{0}\right)$ & 600 & 300 & 15 & 1000 \\
\hline
\end{tabular}

- Nonlinear dispersion: $\chi_{0}^{(3)}=7 \times 10^{-2}(\mathrm{~V} / \mathrm{m})^{-2}, \alpha=0.7$, $\tau_{1}=12.2 \mathrm{fs}, \tau_{2}=32 \mathrm{fs}$.

Figure 3 depicts the results of the dispersive and nonlinear dispersive SF-FDTD computations. In Fig. 3(a), the computed pulse propagating from left to right in a linear Lorentz dispersive medium is shown at $n=2000$ and 4000 steps. A broadening of the computed pulse along the material can be seen with a reduction in its amplitude and carrier frequency modulation due to the linear dispersion.

Figure 3(b) illustrates the situation in which there is dispersive nonlinearity. As can be seen in the figure, a temporal soliton is formed, and the amplitude and width of the main pulse remain essentially unchanged. The transient third-harmonic generation can be identified as a small-amplitude precursor pulse. Figure 3(c) shows the pulse propagation when the Raman effect is taken into account. The Raman contribution is based on a retarded response of the nuclei of the atoms, which is approximated by the response function of damped oscillation having a characteristic frequency $1 / \tau_{1}$ and a damping time constant $\tau_{2}$. Thus, the dispersion in this case is stronger than in Fig. 3(b).

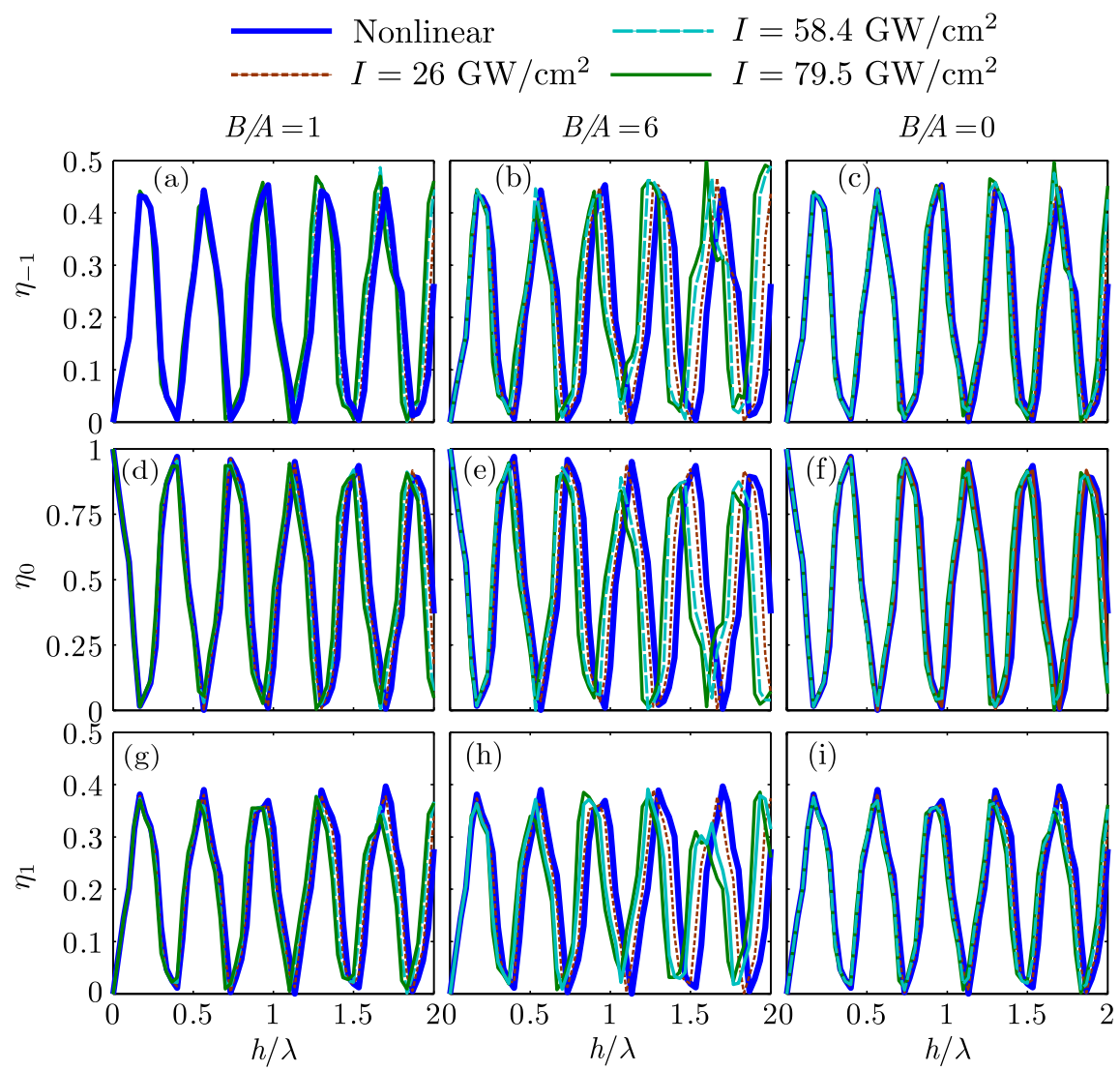

Fig. 5. Transmission diffraction efficiencies as a function of the normalized depth of the pillars. The minus first, zeroth, and first-orders are represented in the first (a)-(c), second (d)-(f), and third (g)-(i) rows of the graphs, respectively. Each column of graphs belongs to a different value of the ratio $B / A$. (a), (d), (g) $B / A=1$. (b), (e), (h) $B / A=6$. (c), (f), (i) $B / A=0$. 
The frequency spectra of the input and output pulses, after they have traveled some distance in the nonlinear medium, are represented in Fig. 4. As expected, in the case of linear dispersion, the spectrum remains almost unchanged. On the contrary, there is a shift and a sharpening of the spectrum due to Raman effects. Moreover, there is a gain due to the Kerr effect. These results reproduce those reported in the literature $[30,32,35]$. In all cases, we assume a plane-wave illumination, instead of a hyperbolic-secant transverse profile normally considered in this type of simulations. This factor may influence the amplitude of the precursor pulse, which differs from the results described in the literature since the latter are not obtained considering an infinite plane of incidence.

\section{Third-Order Nonlinear Media}

Finally, we consider the full tensorial approach applied to third-order nonlinear media. We study the diffraction efficiencies of binary dielectric gratings with an oblique angle of incidence as a function of the thickness of the pillars. The input intensity is increased to trigger the nonlinear effect, and also the ratio $B / A$ is modified based on the phenomena detailed in Subsection 2.D.

The parameters of the SF-FDTD method are summarized in Table 2 . The period of the grating is fixed at $\Lambda / \lambda=20$, and the materials considered for the binary phase grating are the following.

- Pillar material: GaAs with $n_{0}=3.47$ and $A=1.4 \times 10^{-18}(\mathrm{~V} / \mathrm{m})^{2}$.

- Substrate: fused silica with $n_{0}=1.47$.

The diffraction efficiencies for the binary phase grating with nonlinear material in the pillars are shown in Fig. 5. The zero and first diffraction orders are represented as a function of the thickness of the nonlinear material for different physical mechanisms of the third-order nonlinear material. As can be seen, the most relevant discrepancies between the linear and nonlinear curves are found when $B / A=6$. In this case, at higher values of input intensity, the shift of the sidelobes can easily be identified with a considerable modification in the shape of the diffraction efficiencies. In all cases, the nonlinear effects arise when the thickness of

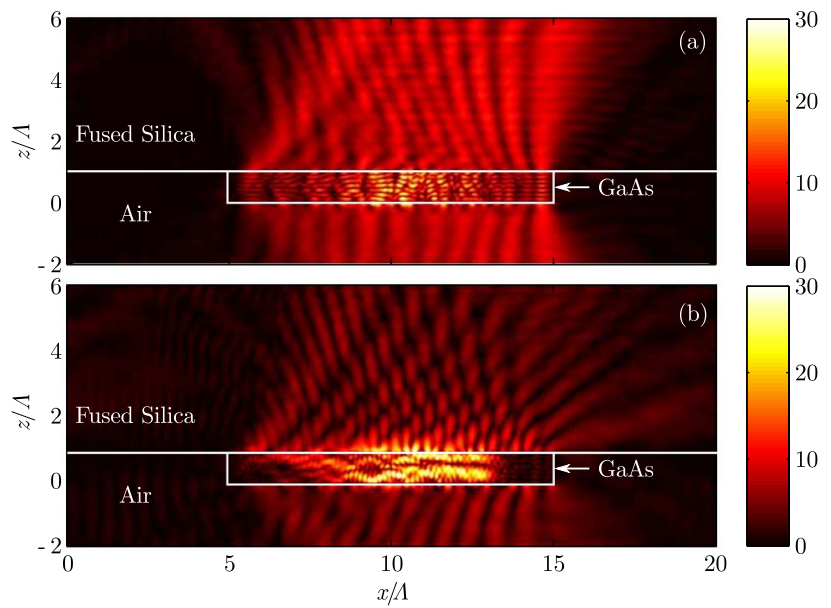

Fig. 6. Differences between electric field components for $B / A=1$ and $B / A=0$ in $\mathrm{GV} / \mathrm{cm}$. Parameters: $\Lambda / \lambda=20$ and $h / \lambda=1$, and input intensity $79.5 \mathrm{GW} / \mathrm{cm}^{2}$. (a) Absolute difference between $E_{x}$ components. (b) Absolute difference between $E_{z}$ components. the pillars increases, since the quantity of nonlinear material is increased.

In order to exemplify the differences that are also found in the cases $B / A=1$ and $B / A=0$, the electric field components in the $x$ and $z$ directions are represented in Fig. 6 . Specifically, the absolute difference between each component is represented for the same structures and time steps but with different $B / A$ values.

\section{CONCLUSIONS}

In this paper, we extended the SF-FDTD method to periodic optical media with second- and third-order nonlinear materials. The nonlinear dispersive behavior in the third-order susceptibility was also considered by means of the Raman contribution. Compared with the previous analysis in [36], the third-order scheme is extended to full tensorial description. This enables a wide range of nonlinear periodic devices to be modeled accurately, which is particularly important in numerous areas of nonlinear optics. In this method, the third-order susceptibility is included using the concept of polarization current, which involves solving a nonlinear system of equations. Hence a fixed-point iterative procedure was adapted to each scenario in order to compute the components of the transformed split-field variables related to the electric field.

We validated the method numerically by comparisons to already-known structures and phenomena, including SHG and temporal solitons in a homogeneous medium. We then showed the efficiency of the method by quantitatively analyzing the diffraction efficiency of binary phase gratings with a tensor-form third-order susceptibility in the grating pillars at oblique incidence.

In general, all finite-difference approaches are computationally intensive. In this paper, the nonlinear system of equations requires an additional iterative process in each SF-FDTD time step to update the electric field, which increases the time and resources required. Thus, the authors are considering including numerical acceleration strategies based on multicore CPUs and GPU computing in order to extend this scheme to two-dimensional nonlinear periodic structures.

\section{ACKNOWLEDGMENTS}

The authors thank Jari Turunen and Yuri Svirko for their useful and constructive comments. The work is partially supported by the Academy of Finland (project 118951), the Strategic Funding of the University of Eastern Finland, the "Ministerio de Economía y Competitividad" of Spain under project FIS2011-29803-C02-01, and the "Generalitat Valenciana" of Spain under projects PROMETEO/2011/021 and ISIC/2012/013.

\section{REFERENCES}

1. R. W. Boyd, Nonlinear Optics, 2nd ed. (Academic, 2003), pp. 161-224

2. G. P. Agrawal, Nonlinear Fiber Optics, 5th ed. (Academic, 2012) 457-492.

3. M. Qasymeh, M. Cada, and S. A. Ponomarenko, "Quadratic electro-optic Kerr effect: applications to photonic devices," IEEE J. Quantum Electron. 44, 740-746 (2008).

4. P. S. Balourdos, D. J. Frantzeskakis, M. C. Tsilis, and I. G. Tigelis, "Reflectivity of a nonlinear discontinuity in optical waveguides," Pure Appl. Opt. 7, 1-11 (1998). 
5. W. D. Deering and M. I. Molina, "Power switching in hybrid coherent couplers," IEEE J. Quantum Electron. 33, 336-340 (1997).

6. Y. Wang, "Nonlinear optical limiter and digital optical switch by cascaded nonlinear couplers: analysis," J. Lightwave Technol. 17, 292-297 (1999).

7. L. Brzozowski and E. H. Sargent, "Optical signal processing using nonlinear distributed feedback structures,” IEEE J. Quantum Electron. 36, 550-555 (2000).

8. S. I. Bozhevolny, K. Pedersen, T. Skettrup, X. Zhang, and M. Belmonte, "Far- and near-field second-harmonic imaging of ferroelectric domain walls," Opt. Commun. 152, 221-224 (1998).

9. I. I. Smolyaninov, H. Y. Liang, C. H. Lee, C. C. Davis, V. Nagarajan, and R. Ramesh, "Near-field second harmonic imaging of the $\mathrm{c} / \mathrm{a} / \mathrm{c} / \mathrm{a}$ polydomain structure of epitaxial $\mathrm{pbzr}_{x} \mathrm{ti}_{1-x} \mathrm{O}_{3}$ thin films," J. Microsc. 202, 250-254 (2001).

10. A. V. Zayats, T. Kalkbrenner, V. Sandoghdar, and J. Mlynek, "Second harmonic generation from individual surface defects under local excitation,” Phys. Rev. B 61, 4545-4548 (2000).

11. S. Takahashi and A. V. Zayats, "Near-field second-harmonic generation at a metal tip apex," Appl. Phys. Lett 80, 3479-3481 (2002).

12. F. Zhou, Y. Liu, Z.-Y. Li, and Y. Xia, "Analytical model for optical bistability in nonlinear metal nano-antennae involving kerr materials," Opt. Express 18, 13337-13344 (2010).

13. S. M. Wang and L. Gao, "Nonlinear responses of the periodic structure composed of single negative materials," Opt. Commun. 267, 197-204 (2006)

14. S. M. Wang, C. J. Tang, T. Pan, and L. Gao, "Bistability and gap soliton in one-dimensional photonic crystal containing singlenegative materials," Phys. Lett. A 348, 424-431 (2006).

15. D. Gao and L. Gao, "Goos-Hänchen shift of the reflection from nonlinear nanocomposites with electric field tunability,” Appl. Phys. Lett. 97, 041903 (2010).

16. W. T. Dong, L. Gao, and C. W. Qiu, "Goos-Hänchen shift at the surface of chiral negative refractive media," Progress Electromagn. Res. 90, 255-268 (2009).

17. J. Yuan and J. Yang, "Computational design for efficient secondharmonic generation in nonlinear photonic crystals,” J. Opt. Soc. Am. B 30, 205-210 (2013).

18. L.-C. Zhao and J. Liu, "Localized nonlinear waves in a two-mode nonlinear fiber,” J. Opt. Soc. Am. B 29, 3119-3127 (2012).

19. K. S. Yee, "Numerical solution of initial boundary value problems involving maxwell's equations in isotropic media," IEEE Trans. Antennas Propag. 14, 302-307 (1966).

20. J. Roden, S. D. Gedney, M. P. Kesler, J. G. Maloney, and P. H. Harms, "Time-domain analysis of periodic structures at oblique incidence: orthogonal and nonorthogonal FDTD implementations," IEEE Trans. Microwave Theor. Tech. 46, 420-427 (1998).

21. J. G. Maloney and M. P. Kesler, "Analysis of antenna arrays using the split-field update FDTD method," in IEEE Antennas and Propagation Society International Symposium (IEEE, 1998), Vol. 4, pp. 420-427.

22. S. M. Amjadi and M. Soleimani, "Design of band-pass waveguide filter using frequency selective surfaces loaded with surface mount capacitors based on split-field update FDTD method," Progress Electromagn. Res. B 43, 271-281 (2008).

23. A. Belkhir and F. I. Baida, "Three-dimensional finite-difference time-domain algorithm for oblique incidence with adaptation of perfectly matched layers and nonuniform meshing: application to the study of a radar dome," Phys. Rev. E 77, 056701 (2008).

24. C. Oh and M. J. Escuti, "Time-domain analysis of periodic anisotropic media at oblique incidence: An efficient FDTD implementation," Opt. Express 14, 11870-11884 (2006).

25. F. I. Baida and A. Belkhir, "Split-field FDTD method for oblique incidence study of periodic dispersive metallic structures," Opt. Lett. 34, 2453-2455 (2009).

26. A. Belkhir, O. Arar, S. S. Benabbes, O. Lamrous, and F. I. Baida, "Implementation of dispersion models in the split-field-finitedifference- time-domain algorithm for the study of metallic periodic structures at oblique incidence," Phys. Rev. E 81, 046705 (2010)
27. A. Shahmansouri and B. Rashidian, "Comprehensive threedimensional split-field finite-difference time-domain method for analysis of periodic plasmonic nanostructures: near- and far-field formulation,” J. Opt. Soc. Am. B 28, 2690-2700 (2011).

28. A. Shahmansouri and B. Rashidian, "GPU implementation of split-field finite-difference time-domain method for DrudeLorentz dispersive media," Progress Electromagn. Res. 125, 55-77 (2012)

29. A. Taflove and S. C. Hagness, Computational Electrodynamics: The Finite-Difference Time-Domain Method, 3rd ed. (Artech House, 2005), pp. 353-406.

30. R. M. Joseph and A. Taflove, "FDTD maxwell's equations models for nonlinear electrodynamics and optics,” IEEE Trans. Antennas Propag. 45, 364-374 (1997).

31. P. M. Goorjian, A. Taflove, R. M. Joseph, and S. C. Hagness, "Computational modeling of femtosecond optical solitons from Maxwell's equations,” IEEE J. Quantum Electron. 28, 2416-2422 (1992).

32. P. M. Goorjian and A. Taflove, "Direct time integration of maxwell's equations in nonlinear dispersive media for propagation and scattering of femtosecond electromagnetic solitons," Opt. Lett. 17, 180-182 (1992).

33. K. H. Lee, I. Ahmed, R. S. M. Goh, E. H. Khoo, E. P. Li, and T. G. G. Hung, "Implementation of the FDTD method based on Lorentz-Drude dispersive model on GPU for plasmonics applications," Progress Electromagn. Res. 116, 441-456 (2011)

34. Y.-Q. Zhang and D. B. Ge, "A unified FDTD approach for electromagnetic analysis of dispersive objects," Progress Electromagn. Res. 96, 155-172 (2009)

35. M. Fujii, M. Tahara, I. Sakagami, W. Freude, and P. Russer "High-order FDTD and auxiliary differential equation formulation of optical pulse propagation," IEEE J. Quantum Electron. 40, 175-182 (2004).

36. J. Francés, J. Tervo, and C. Neipp, "Split-field finite-difference time-domain scheme for Kerr-type nonlinear periodic media," Progress Electromagn. Res. 134, 559-579 (2013).

37. S. D. Gedney, "An anisotropic perfectly matched layer-absorbing medium for the truncation of FDTD lattices," IEEE Trans Antennas Propag. 44, 1630-1639 (1996).

38. G. Zheng, A. A. Kishk, A. W. Glisson, and A. B. Yakovlev, "Implementation of Mur's absorbing boundaries with periodic structures to speed up the design process using finite-difference time-domain method," Progress Electromagn. Res. 58, 101-114 (2006).

39. P. Butcher and D. Cotter, The Elements of Nonlinear Optics (Cambridge University, 1990), pp. 12-149.

40. H. M. Al-Mudhaffar, M. A. Alsunaidi, and H. M. Masoudi, "Fullwave solution of the second harmonic generation problem using a nonlinear FDTD algorithm," in Progress in Electromagnics Research Symposium Proceedings, Prague, Czech Repulic (2007), pp. 479-482.

41. M. A. Alsunaidi, H. M. Al-Mudhaffar, and H. M. Masoudi, "Vectorial FDTD technique for the analysis of optical secondharmonic generation," IEEE Photon. Technol. Lett. 5, 310-312 (2009).

42. M. A. Alsunaidi and F. S. Al-Hajiri, "Efficient NL-FDTD solution schemes for the phase-sensitive second harmonic generation problem,” J. Lightwave Technol. 27, 4964-4969 (2009).

43. C. Dissanayake, M. Premaratne, I. D. Rukhlenko, and G. P. Agrawal, "FDTD modeling of anisotropic nonlinear optical phenomena in silicon waveguides," Opt. Express 18, 21427-21448 (2010).

44. M. Ammann, "Non-trivial materials in EM-FDTD," Master's thesis (Department of Physics, Swiss Federal Institute of Technology, 2007).

45. J. H. Greene and A. Taflove, "General vector auxiliary differential equation finite-difference time-domain method for nonlinear optics," Opt. Express 14, 8305-8310 (2006).

46. S. Malaguti, A. Armaroli, G. Bellanca, S. Trillo, S. Combrié, P. Colman, and A. De Rossi, "Temporal dynamics of nonlinear switching in GaAs photonoic-crystal-based devices," in 36th European Conference and Exhibition on Optical Communication (ECOC) (IEEE, 2010), pp. 1-3. 